\title{
Seasonal Stability in the Microbiomes of Temperate Gorgonians and the Red Coral Corallium rubrum Across the Mediterranean Sea
}

\author{
Jeroen A. J. M. van de Water $^{1}$ (D) Christian R. Voolstra ${ }^{2} \cdot$ Cecile Rottier $^{1}$ • \\ Silvia Cocito $^{3}$ - Andrea Peirano ${ }^{3}$ - Denis Allemand ${ }^{1} \cdot$ Christine Ferrier-Pagès $^{1}$
}

\begin{abstract}
Populations of key benthic habitat-forming octocoral species have declined significantly in the Mediterranean Sea due to mass mortality events caused by microbial disease outbreaks linked to high summer seawater temperatures. Recently, we showed that the microbial communities of these octocorals are relatively structured; however, our knowledge on the seasonal dynamics of these microbiomes is still limited. To investigate their seasonal stability, we collected four soft gorgonian species (Eunicella singularis, Eunicella cavolini, Eunicella verrucosa and Leptogorgia sarmentosa) and the precious red coral (Corallium rubrum) from two coastal locations with different terrestrial impact levels in the Mediterranean Sea, and used next-generation amplicon sequencing of the 16S rRNA gene. The microbiomes of all soft gorgonian species were dominated by the same 'core microbiome' bacteria belonging to the Endozoicomonas and the Cellvibrionales clade BD1-7, whereas the red coral microbiome was primarily composed of 'core' Spirochaetes, Oceanospirillales ME2 and Parcubacteria. The associations with these bacterial taxa were
\end{abstract}

Jeroen A. J. M. van de Water jvdewater@centrescientifique.mc

1 Centre Scientifique de Monaco, 8 Quai Antoine 1er, 98000 Monaco, Monaco

2 Red Sea Research Center, Division of Biological and Environmental Science and Engineering (BESE), King Abdullah University of Science and Technology (KAUST), Thuwal 23955-6900, Saudi Arabia

3 Marine Environment Research Centre, ENEA, La Spezia, Italy relatively consistent over time at each location for each octocoral species. However, differences in microbiome composition and seasonal dynamics were observed between locations and could primarily be attributed to locally variant bacteria. Overall, our data provide further evidence of the intricate symbiotic relationships that exist between Mediterranean octocorals and their associated microbes, which are ancient and highly conserved over both space and time, and suggest regulation of the microbiome composition by the host, depending on local conditions.

Keywords Endozoicomonas · Spirochaetes · Red coral · Gorgonian · 16S rRNA gene $\cdot$ Bacterial community · Holobiont $\cdot$ Evolution $\cdot$ Microbiome $\cdot$ Symbiosis

\section{Introduction}

The 'holobiont' is a collective term for the inter-kingdom assemblages of host organisms and their microbial symbionts, including bacteria, archaea, viruses, fungi and algae. Bacterial symbioses have been recognized as essential for the health of eukaryotes and are integral to their adaptation and acclimation potential to environmental change $[1,2]$. Increasing research efforts have endeavoured to identify the specific and obligate symbioses between prokaryotes and corals, among the thousands of bacterial phylotypes associated with individual coral colonies [3]. Recent studies have shown that the coral holobiont contains a 'core microbiome' [4, 5], consisting of microbes that are consistently found within a species, as well as microbes whose presence depends on local conditions, and transient microbial associates [6, 7]. While the functional roles of the cnidarian-associated microbiota are still largely unknown, they have been implicated in many services beneficial to the host, such as nitrogen fixation [8,9], sulphur-cycling 
[10] and microbiome regulation through the secretion of antibiotics [11-14] and exclusion of pathogens by occupying available microbial niches $[15,16]$. As such, it is essential for holobiont fitness to maintain a stable multi-functional microbial community. In contrast to the highly diverse microbiomes of tropical corals, Mediterranean octocorals have been shown to harbour highly structured bacterial assemblages dominated for $>90 \%$ by only a few species $[17,18]$, making them ideal model organisms to study cnidarianmicrobe interactions.

In the Mediterranean Sea, gorgonians are the most important habitat-forming species of benthic communities, contributing significantly to the structural complexity, biomass and biodiversity of these ecosystems [19]. The success of gorgonians can at least in part be attributed to the specific symbioses with bacteria, which were recently found to be relatively stable across spatial scales $[7,18]$. In species belonging to the Gorgoniidae family, bacteria from the genus Endozoicomonas have been found to be the most prevalent [20-23], with some lesser contributions from clade BD1-7 [7]. Endozoicomonas have been found in numerous marine invertebrates and have been identified as crucial to the health of corals (expertly reviewed in [24]), with a loss of these bacteria having a severe negative impact on holobiont functioning. In tropical octocorals and deep-sea gorgonians, especially the precious red coral Corallium rubrum, Spirochaetes may be one of the (co-)dominant microbial associates [18, 25, 26], which may play roles in nitrogen and carbon fixation, as seen in the Spirochaete-termite symbiosis $[27,28]$. The spatial stability of these bacteria-host associations, which may reside in the same habitat and location, suggests strong selection mechanisms employed by the holobiont of Mediterranean gorgonians. Indeed, gorgonian tissue extracts are known to interfere with bacterial quorum-sensing and have potent antimicrobial activity [29-32]. Local disturbances and elevated seawater temperatures have, however, been linked to disruptions in the microbiome of gorgonians [7, 33]. Recent mass mortality events caused by microbial disease outbreaks and thermal anomalies have resulted in significant population declines $[34,35]$. This suggests that shifts in the gorgonian microbiome towards an 'unhealthy', dysbiotic state could occur on a seasonal scale, particularly during the summer months. Mediterranean octocorals experience large variations in seawater temperatures, from a minimum of $12{ }^{\circ} \mathrm{C}$ in winter to at least $23{ }^{\circ} \mathrm{C}$ in summer [36] and may experience significant river discharges, which brings sediment, turbidity and pollution, following rain in coastal areas, particularly in spring and autumn. It is therefore important to understand the seasonal variability in the gorgonian-associated bacterial assemblages or determine whether it is as stable as across spatial scales.

Here, we describe the temporal patterns in the microbiome of five closely (genera Eunicella and Leptogorgia) and distantly (genus Corallium) related octocorals from the
Mediterranean Sea at a clear and turbid location. We show (1) that the microbiomes associated with octocorals are distinct from the microbial communities in the surrounding seawater and (2) that the highly structured bacterial assemblages are stable over time, but that local conditions may influence microbial community composition. Overall, our results provide new insights into the unique, specific associations between gorgonians and their microbial symbionts.

\section{Material and Methods}

\section{Study Species and Sampling Regime}

Octocoral samples were collected at 30-40 m depth near Cassis, France $\left(43^{\circ} 12^{\prime} \mathrm{N} 05^{\circ} 28^{\prime} \mathrm{E}\right)$ and at 16-20 $\mathrm{m}$ depth on Dante Shoal near La Spezia, Italy $\left(44^{\circ} 01^{\prime} \mathrm{N} 09^{\circ} 50^{\prime} \mathrm{E}\right)$ every 3 months ( $\pm 1-2$ weeks) between May 2014 and July 2015 (Fig. 1; Table 1). The La Spezia sampling location is characterized by high turbidity, which is highest in springsummer and the lowest in fall-winter (total suspended solids concentration ranging between 1.61 and $2.65 \mathrm{mg} \mathrm{L}^{-1}$ [37]) and is the result of terrestrial runoff from the Magra River and sewage from the city of La Spezia, both of which get caught up in the westward Ligurian current [38]. In contrast, the sampling location near Cassis is not impacted by terrestrial runoff, as no river is present within a $60 \mathrm{~km}$ radius, and sewage input from the town of $\sim 7000$ inhabitants is limited, corresponding with low turbidity levels. While Eunicella singularis, Eunicella verrucosa and Leptogorgia sarmentosa were sampled at both locations; samples of Corallium rubrum and Eunicella cavolini were only obtained near Cassis as these species were not present at the La Spezia location. Fragments of five visually healthy colonies that did not show any macroscopic signs of epibiosis or partial mortality, were collected at each location and time point, with the exception of July 2015 when only three replicate fragments were collected at Cassis. Samples were rinsed twice with $0.2-\mu \mathrm{m}$ filtered seawater to remove exogenous, loosely associated microorganisms and stored in ice-cold RNAlater (ThermoFisher Scientific) at $4{ }^{\circ} \mathrm{C}$. In addition, three samples of 21 of seawater each were collected next to the octocoral colonies and filtered sequentially through 8,3 and $0.2-\mu \mathrm{m}$ Whatman Nuclepore Track-Etched filters (Sigma-Aldrich), and the retentate was kept in RNAlater at $4{ }^{\circ} \mathrm{C}$.

\section{DNA Extraction and 16S rRNA Gene Amplicon Library Preparation}

To extract DNA from tissues and seawater filter retentate, we used the Genomic DNA buffer set and Genomic-tip 20/G columns (QIAGEN, Hilden, Germany) following the manufacturer's sample preparation and lysis protocol for tissues- 


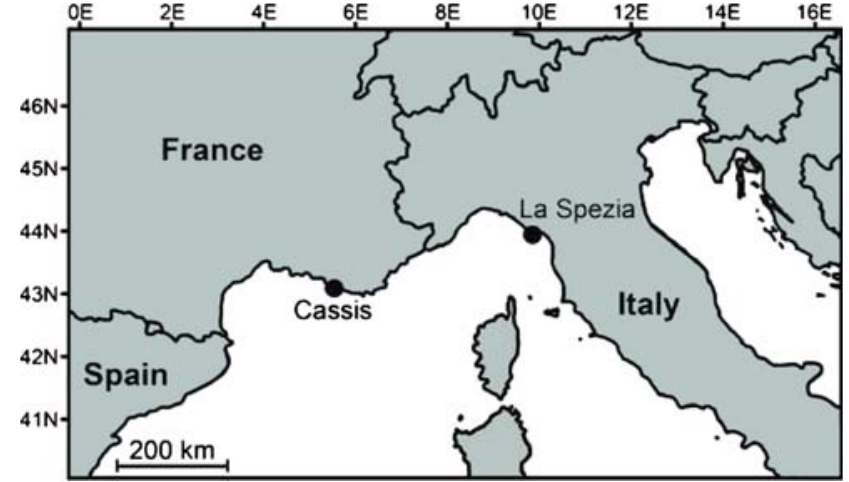

Fig. 1 Overview of the sampling locations. Gorgonians were collected from the Western Mediterranean Sea at two locations: Cassis in France and La Spezia in Italy

octocoral fragments and filters were placed directly in lysis buffer. For each soft gorgonian sample, 16S rDNA amplicon libraries were generated in triplicate using the Multiplex PCR kit (QIAGEN, Hilden, Germany) and the 784F/1061R primer set [39] in 30- $\mu \mathrm{l}$ reaction volumes containing $0.2 \mu \mathrm{M}$ of each primer and $150 \mathrm{ng}$ of template DNA. The PCR protocol consisted of an initial denaturation step of $95^{\circ} \mathrm{C}$ for $15 \mathrm{~min}$ followed by 30 amplification cycles (denaturation at $95^{\circ} \mathrm{C}$ for $30 \mathrm{~s}$, annealing at $55^{\circ} \mathrm{C}$ for $40 \mathrm{~s}$ and extension at $72{ }^{\circ} \mathrm{C}$ for $40 \mathrm{~s}$ ) and a final extension step of $10 \mathrm{~min}$ at $72{ }^{\circ} \mathrm{C}$. PCR products were cleaned using the PureLink PCR purification kit (Invitrogen, Carlsbad CA, USA) and subsequently quantified using a Qbit fluorometer (Invitrogen, Carlsbad CA, USA) and ran on $1 \%$ agarose electrophoresis gel to confirm purity. PCR product triplicates were pooled in equal quantity and sent to the KAUST BioScience Core Laboratory (Thuwal, Saudi Arabia). Libraries were generated using the Nextera XT DNA Library Prep Kit (Illumina, San Diego, CA) and sequenced on the Illumina MiSeq platform.

To allow for direct comparisons with our previous study on spatial patterns in the C. rubrum microbiome, sequencing libraries of the red coral DNA samples were prepared with the $8 \mathrm{~F} / 334 \mathrm{R}$ primer set targeting the $\mathrm{V} 1 / \mathrm{V} 2$ regions of the $16 \mathrm{~S}$ rRNA gene [40] and were sequenced on the Illumina MiSeq platform at the Molecular Research LP laboratory (Shallowater, Texas, USA).

\section{S rRNA Gene Amplicon Data Analysis}

The QIIME pipeline [41] was used for data processing. Paired-end MiSeq sequencing of the 176 samples of gorgonians generated 24,131,112 reads. Forward and reverse reads were merged using SeqPrep (https:/github.com/jstjohn/ SeqPrep), producing 9,539,086 joined reads (78.5\%). Primer sequences were removed using extract_barcodes.py and the split_libraries.py script was used to remove low quality (Phred $<20$ ) sequences, reads <200 bp in length, and to assign each read to its respective sample. The resulting file was checked for chimeric sequences against the SILVA v123 database [42] using UCHIME [43]. The final quality-filtered sequence file contained 9,230,759 reads with a mean read length of $257 \pm 8$ base pairs. Operational taxonomic units (OTUs) were defined at the level of $97 \%$ similarity followed by taxonomy assignments against the SILVA reference database (version 123) using the UCLUST algorithm [44]. Singletons, unassigned OTUs, and OTUs classified as chloroplast or mitochondria were removed from the dataset. The OTU table was rarefied to 15,587 reads for comparisons across species, locations and time points. Alpha diversity metrics (total observed number of OTUs, predicted species (chao1), Simpson's evenness, Shannon-Wiener diversity and Fisher's alpha) were generated from OTU tables using the QIIME pipeline. In addition, the overall gorgonian core microbiome as well as the core microbiome of each species and the communities of locally stable bacterial associates were analysed. The complete dataset has been deposited in the NCBI Sequence Read Archive (SRA) with accession number SRP107866.
Table 1 Overview of the number of samples collected per species and of seawater at each location and sampling time point

\begin{tabular}{|c|c|c|c|c|c|c|}
\hline \multirow[b]{2}{*}{ Location } & \multirow[b]{2}{*}{ Species/seawater } & \multicolumn{3}{|l|}{2014} & \multicolumn{2}{|l|}{2015} \\
\hline & & Spring & Summer & Winter & Early spring & Late spring \\
\hline \multirow[t]{6}{*}{ Cassis } & Corallium rubrum & 2 & 5 & 5 & 5 & 3 \\
\hline & Eunicella cavolini & 5 & 5 & 5 & 5 & 3 \\
\hline & Eunicella singularis & 5 & 5 & 5 & 5 & 3 \\
\hline & Eunicella verrucosa & 5 & 5 & 5 & 5 & 3 \\
\hline & Leptogorgia sarmentosa & 5 & 5 & 5 & 5 & 3 \\
\hline & Seawater & 3 & 3 & 3 & 3 & 3 \\
\hline \multirow[t]{4}{*}{ La Spezia } & Eunicella singularis & 5 & 5 & 5 & 5 & \\
\hline & Eunicella verrucosa & 5 & 5 & 5 & 5 & \\
\hline & Leptogorgia sarmentosa & 5 & 5 & 5 & 5 & \\
\hline & Seawater & 3 & 3 & 3 & 3 & \\
\hline
\end{tabular}


Sequencing data from MR DNA (Shallowater, Texas, USA) was obtained as pre-merged and re-oriented sequence .fasta files and their associated quality .qual files. Data files contained 3,845,860 paired-end reads, which were quality filtered and chimera checked producing 3,280,553 reads with a mean length of $322 \pm 15$ base pairs. Quality filtering, OTU picking, taxonomy assignments and subsequent analyses were conducted as described above. This dataset has been deposited under accession number SRP107893 in the NCBI SRA.

\section{Statistical Analysis}

The phyloseq package [45] integrated in $R$ was used to generate statistically relevant graphical presentations of the microbiome data obtained. To visualise differences between gorgonian-associated bacterial communities, non-metric dimensional scaling (nMDS) on Bray-Curtis dissimilarity matrices was performed. Ellipses representing the $95 \%$ confidence intervals were added to nMDS plots if $>3$ biological replicates were obtained. A heatmap was generated to present the average abundances of core microbiome OTUs within the holobiont of each gorgonian species at both locations over the different time points. Permutational analysis of variance (permANOVA) and pair-wise comparisons, with Monte Carlo simulations conducted in parallel, were performed under Type III partial sums of squares and 9999 permutations under the reduced model to test for differences in the beta diversity (based on the Bray-Curtis dissimilarity matrices) among bacterial communities over time at the two different locations for each gorgonian host species. To test for temporal variations in the microbial communities near Cassis, five sampling 'time' factor levels were considered and four levels at $\mathrm{La}$ Spezia. To assess differences in community diversity between locations at each sampling time point, comparisons were only performed for the four seasons, where samples had been collected at both locations. To provide an overall assessment of differences in the alpha diversity of the microbiomes, permANOVA and pair-wise comparisons were conducted (with the same settings as described above) based on a Euclidean distance matrix. The matrix was generated by considering all alpha diversity metrics as outcome variables, square root transformation of the data, and subsequent calculation of Euclidean distances between samples. All diversity analyses were conducted using PRIMER 6 and PERMANOVA+ (PRIMER-E Ltd) and differences were considered significant when $p<0.05$. Differential abundance analysis was performed to investigate which OTUs were responsible for the observed differences in microbiome composition using negative binomial modelling (likelihood ratio tests to test for factors significantly impacting microbiome composition and Wald tests for pair-wise comparisons) using the DESeq2 package in $R$ [46].

\section{Nucleotide Sequence Accession Numbers}

The sequences of OTUs belonging to the core microbiome and locally stable microbial associate communities found in this study were deposited in the GenBank database under accession numbers MF148323-MF148422.

\section{Results}

\section{Assessment of the Bacterial Diversity in Soft Gorgonians}

Consistent with earlier reports [7, 18], the composition of bacterial communities associated with soft gorgonians (Fig. S1A) was significantly different from bacterial communities in seawater at all time points at both sites, i.e. Cassis and La Spezia $(p<0.03$; Suppl. File S1-Table S1 and S2). In general, the temporal stability/differences in the octocoral-associated microbiomes significantly depended on the host species and the sampling location (Supplementary File S1-Table S1). Microbiomes of the different gorgonians were overall speciesspecific, although no major differences were observed (I) between the microbiomes of E. cavolini and E. singularis at Cassis, (II) among any gorgonian species in the late spring of 2015 at Cassis and (III) between E. singularis and E. verrucosa in the summer of 2014 and the winter 2014/2015 at La Spezia (Suppl. File S1-Table S1 and S2). In addition, we found that the bacterial assemblages of $E$. verrucosa and $L$. sarmentosa differed significantly between the two locations at all time points, while such differences were observed for $E$. singularis only in the winter 2014/2015 and early spring of 2015 (Fig. 2; Suppl. File S1-Table S3). On temporal scales, however, the beta diversity of the microbiomes of all gorgonians was relatively stable at Cassis, with the only significant difference observed between the spring of 2014 and winter 2014/2015 in both E. singularis and L. sarmentosa (Fig. 2; Suppl. File S1-Table S4). In La Spezia, no significant temporal differences were observed in the microbiome diversity of Eunicella spp., but significant microbiome changes were observed in $L$. sarmentosa between spring sampling time points and the summer and winter time points (Fig. 2; Suppl. File S1-Table S4). In contrast to the differences in beta diversity, no major differences in alpha diversity were observed between gorgonian host species, but the alpha diversity in seawater was generally higher than in gorgonian microbiomes (Table 2; Suppl. File S1-Table S2).

\section{Composition of the Gorgonian Microbiome}

The microbiome of all soft gorgonians was largely dominated by Gammaproteobacteria and Alphaproteobacteria (Suppl. File $S$ 2). Bacteria belonging to other taxonomic classes, including Mollicutes, Flavobacteriia, Spirochaetes, Cytophagia and Sphingobacteria contributed to a lesser extend to the 
A

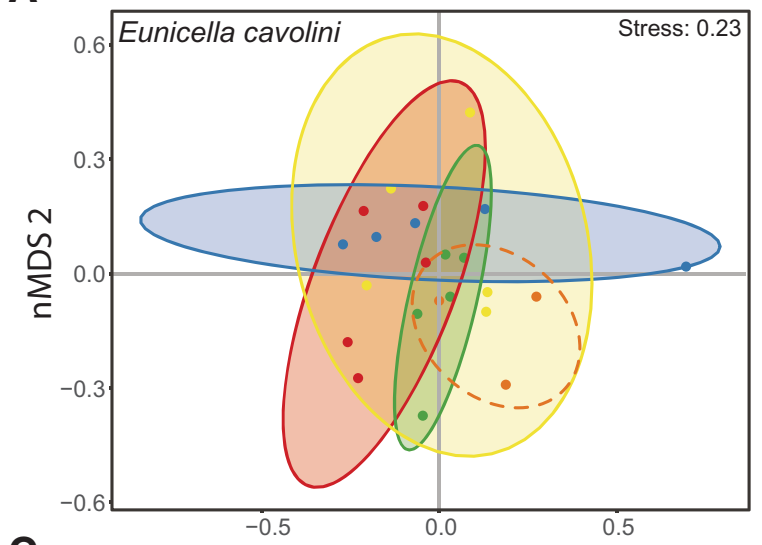

C

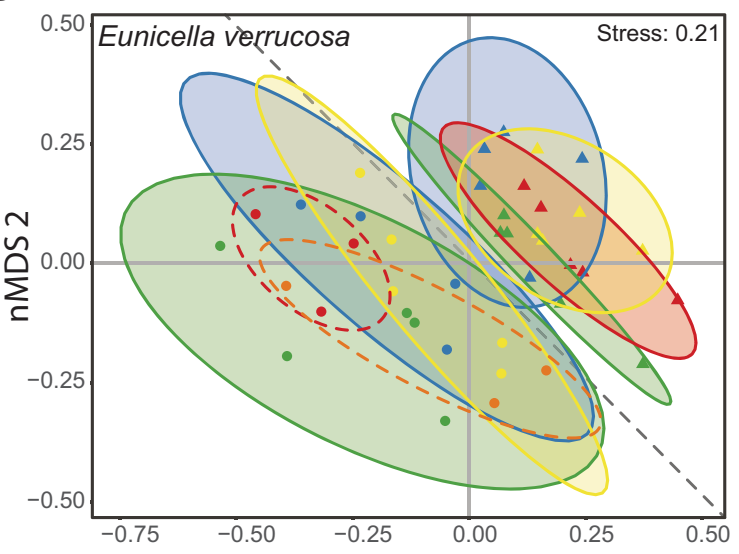

B

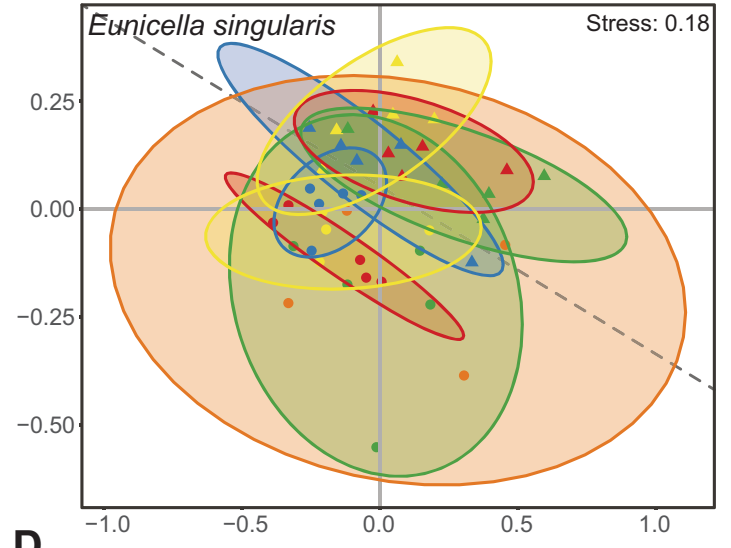

D
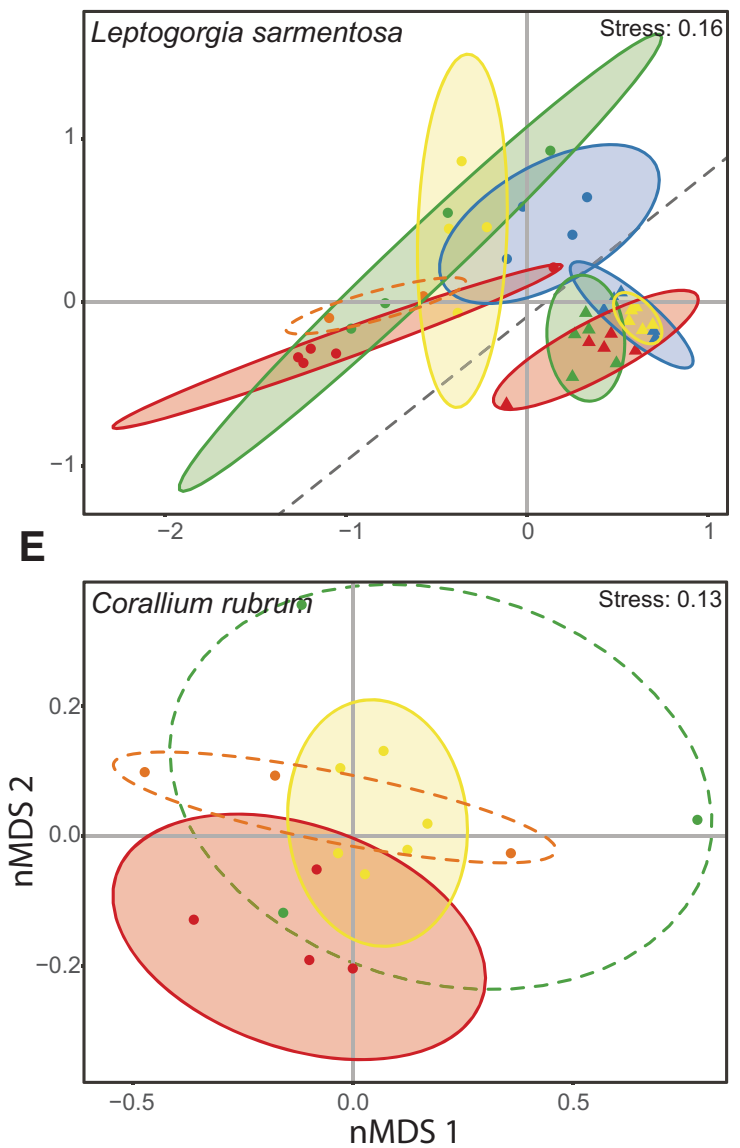

Corallium rubrum. Locations are indicated with symbols, and time points with colour. Ellipses with solid lines indicate the $95 \%$ confidence interval. Ellipses with dotted lines were added for illustrative purpose as sample replicate number was $\leq 3$

dominated the microbiome of all gorgonians, found that three OTUs (Endozoicomonas OTU47380 and OTU69149; BD1-7 OTU20874) were (1) consistently associated with all gorgonian host species across space and time, (2) the main contributors to the gorgonian microbiome and (3) were identical to core microbes previously identified (accession number KU738805, KU738801 and KU738792 [7], respectively) (OTUs present in $100 \%$ of the samples of a species), which pending on host species and time of year (Suppl. File S2). The main Gammaproteobacteria found associated with all gorgonians were bacteria belonging to the genus Endozoicomonas (order Oceanospirillales) and the genus 'clade BD1-7' (order
Cellvibrionales) (Fig. 3). Analysis of the core microbiome
Mediterranean octocorals. a-e Beta diversity of the microbiome is presented in a non-metric dimensional scaling plot based on BrayCurtis dissimilarity matrices for different octocoral species a Eunicella cavolini, $\mathbf{b}$ E. singularis, $\mathbf{c}$ E. verrucosa, $\mathbf{d}$ Leptogorgia sarmentosa and $\mathbf{e}$

gorgonian microbiome, but their abundances did change de-

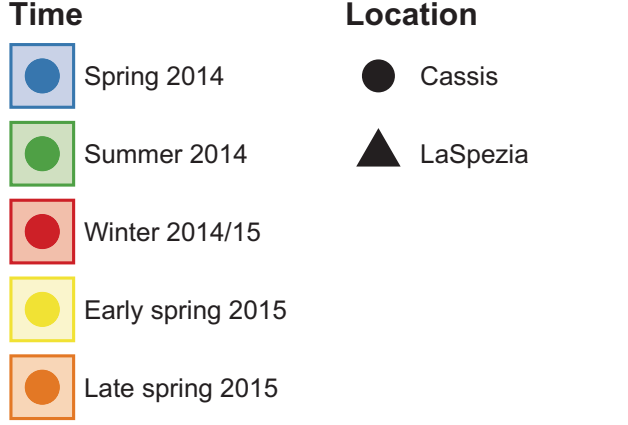


Table 2 Alpha diversity metrics (mean \pm SEM) for samples of five gorgonian species collected at the two different locations throughout the year

\begin{tabular}{|c|c|c|c|c|c|c|c|}
\hline \multirow[b]{2}{*}{ Species } & \multirow[b]{2}{*}{ Location } & \multirow[b]{2}{*}{ Time point } & \multicolumn{5}{|c|}{ Alpha diversity metric } \\
\hline & & & chao1 & Shannon-Wiener index & Simpson's evenness & Fisher's alpha & Unique OTUs \\
\hline \multirow{5}{*}{ E. cavolini } & \multirow{5}{*}{ Cassis } & Spring 2014 & $1088 \pm 84$ & $1.71 \pm 0.46$ & $0.01 \pm 0.00$ & $54.9 \pm 17.2$ & $302 \pm 75$ \\
\hline & & Summer 2014 & $769 \pm 89$ & $2.11 \pm 0.34$ & $0.01 \pm 0.00$ & $44.8 \pm 4.5$ & $261 \pm 22$ \\
\hline & & Winter $2014 / 2015$ & $715 \pm 92$ & $1.21 \pm 0.05$ & $0.01 \pm 0.00$ & $31.6 \pm 2.7$ & $195 \pm 14$ \\
\hline & & Early spring 2015 & $876 \pm 54$ & $2.11 \pm 0.47$ & $0.01 \pm 0.00$ & $48.0 \pm 9.5$ & $275 \pm 46$ \\
\hline & & Late spring 2015 & $836 \pm 93$ & $2.56 \pm 0.37$ & $0.01 \pm 0.00$ & $56.3 \pm 8.4$ & $315 \pm 39$ \\
\hline \multirow[t]{9}{*}{ E. verrucosa } & \multirow[t]{5}{*}{ Cassis } & Spring 2014 & $841 \pm 105$ & $2.33 \pm 0.56$ & $0.01 \pm 0.00$ & $70.9 \pm 15.8$ & $377 \pm 70$ \\
\hline & & Summer 2014 & $746 \pm 117$ & $1.44 \pm 0.39$ & $0.00 \pm 0.00$ & $67.7 \pm 14.6$ & $362 \pm 66$ \\
\hline & & Winter $2014 / 2015$ & $497 \pm 40$ & $0.76 \pm 0.28$ & $0.01 \pm 0.00$ & $37.5 \pm 3.6$ & $226 \pm 18$ \\
\hline & & Early spring 2015 & $871 \pm 115$ & $1.97 \pm 0.50$ & $0.00 \pm 0.00$ & $80.8 \pm 17.2$ & $418 \pm 72$ \\
\hline & & Late spring 2015 & $952 \pm 244$ & $2.32 \pm 1.18$ & $0.01 \pm 0.00$ & $102.5 \pm 34.4$ & $502 \pm 147$ \\
\hline & \multirow[t]{4}{*}{ La Spezia } & Spring 2014 & $884 \pm 176$ & $3.97 \pm 0.50$ & $0.01 \pm 0.00$ & $113.0 \pm 27.1$ & $546 \pm 104$ \\
\hline & & Summer 2014 & $1071 \pm 128$ & $4.53 \pm 0.62$ & $0.01 \pm 0.00$ & $145.2 \pm 17.2$ & $677 \pm 63$ \\
\hline & & Winter 2014/2015 & $1276 \pm 74$ & $4.73 \pm 0.59$ & $0.01 \pm 0.00$ & $174.8 \pm 15.3$ & $784 \pm 54$ \\
\hline & & Early spring 2015 & $1060 \pm 88$ & $3.45 \pm 0.33$ & $0.01 \pm 0.00$ & $104.1 \pm 9.5$ & $521 \pm 38$ \\
\hline \multirow[t]{9}{*}{ L. sarmentosa } & \multirow[t]{5}{*}{ Cassis } & Spring 2014 & $848 \pm 39$ & $3.36 \pm 0.27$ & $0.01 \pm 0.00$ & $75.4 \pm 9.1$ & $401 \pm 40$ \\
\hline & & Summer 2014 & $1334 \pm 493$ & $3.64 \pm 0.87$ & $0.01 \pm 0.00$ & $190.4 \pm 83.2$ & $789 \pm 278$ \\
\hline & & Winter 2014/2015 & $2591 \pm 521$ & $5.91 \pm 1.18$ & $0.01 \pm 0.01$ & $476.0 \pm 118.2$ & $1605 \pm 334$ \\
\hline & & Early spring 2015 & $1140 \pm 175$ & $3.25 \pm 0.32$ & $0.01 \pm 0.00$ & $109.2 \pm 16.6$ & $539 \pm 66$ \\
\hline & & Late spring 2015 & $2146 \pm 550$ & $5.35 \pm 1.78$ & $0.01 \pm 0.01$ & $338.8 \pm 124.7$ & $1282 \pm 361$ \\
\hline & \multirow[t]{4}{*}{ La Spezia } & Spring 2014 & $699 \pm 48$ & $2.58 \pm 0.11$ & $0.01 \pm 0.00$ & $49.3 \pm 3.5$ & $283 \pm 17$ \\
\hline & & Summer 2014 & $973 \pm 60$ & $3.23 \pm 0.21$ & $0.01 \pm 0.00$ & $78.9 \pm 4.3$ & $417 \pm 18$ \\
\hline & & Winter 2014/2015 & $896 \pm 109$ & $3.30 \pm 0.18$ & $0.02 \pm 0.00$ & $67.7 \pm 12.9$ & $364 \pm 57$ \\
\hline & & Early spring 2015 & $717 \pm 29$ & $2.73 \pm 0.12$ & $0.01 \pm 0.00$ & $51.0 \pm 2.9$ & $292 \pm 14$ \\
\hline \multirow[t]{9}{*}{ E. singularis } & \multirow[t]{5}{*}{ Cassis } & Spring 2014 & $826 \pm 99$ & $1.88 \pm 0.22$ & $0.01 \pm 0.00$ & $42.6 \pm 4.6$ & $251 \pm 22$ \\
\hline & & Summer 2014 & $805 \pm 171$ & $2.87 \pm 0.33$ & $0.01 \pm 0.00$ & $65.4 \pm 15.4$ & $351 \pm 70$ \\
\hline & & Winter $2014 / 2015$ & $758 \pm 62$ & $1.96 \pm 0.17$ & $0.01 \pm 0.00$ & $45.8 \pm 8.4$ & $264 \pm 41$ \\
\hline & & Early spring 2015 & $772 \pm 128$ & $2.03 \pm 0.25$ & $0.01 \pm 0.00$ & $51.6 \pm 14.5$ & $290 \pm 65$ \\
\hline & & Late spring 2015 & $808 \pm 121$ & $3.01 \pm 0.95$ & $0.01 \pm 0.00$ & $74.3 \pm 28.3$ & $383 \pm 119$ \\
\hline & \multirow[t]{4}{*}{ La Spezia } & Spring 2014 & $982 \pm 162$ & $2.02 \pm 0.46$ & $0.01 \pm 0.00$ & $63.0 \pm 20.6$ & $336 \pm 88$ \\
\hline & & Summer 2014 & $1146 \pm 121$ & $4.16 \pm 0.76$ & $0.01 \pm 0.00$ & $117.0 \pm 25.4$ & $561 \pm 102$ \\
\hline & & Winter 2014/2015 & $1072 \pm 146$ & $3.11 \pm 0.74$ & $0.01 \pm 0.00$ & $86.5 \pm 23.9$ & $438 \pm 94$ \\
\hline & & Early spring 2015 & $859 \pm 67$ & $1.95 \pm 0.22$ & $0.01 \pm 0.00$ & $48.3 \pm 7.4$ & $277 \pm 35$ \\
\hline \multirow[t]{9}{*}{ Seawater } & \multirow[t]{5}{*}{ Cassis } & Spring 2014 & $2122 \pm 124$ & $6.30 \pm 0.03$ & $0.03 \pm 0.00$ & $225.1 \pm 12.4$ & $956 \pm 40$ \\
\hline & & Summer 2014 & $2172 \pm 140$ & $6.70 \pm 0.05$ & $0.04 \pm 0.00$ & $225.2 \pm 18.4$ & $956 \pm 61$ \\
\hline & & Winter 2014/2015 & $1371 \pm 66$ & $7.07 \pm 0.01$ & $0.08 \pm 0.00$ & $171.7 \pm 5.7$ & $776 \pm 20$ \\
\hline & & Early spring 2015 & $1717 \pm 49$ & $6.68 \pm 0.05$ & $0.05 \pm 0.01$ & $182.6 \pm 4.5$ & $814 \pm 16$ \\
\hline & & Late spring 2015 & $2327 \pm 166$ & $6.77 \pm 0.16$ & $0.04 \pm 0.00$ & $239.5 \pm 28.2$ & $1001 \pm 89$ \\
\hline & \multirow[t]{4}{*}{ La Spezia } & Spring 2014 & $1238 \pm 105$ & $6.38 \pm 0.17$ & $0.04 \pm 0.00$ & $151.9 \pm 16.7$ & $703 \pm 60$ \\
\hline & & Summer 2014 & $1726 \pm 185$ & $6.43 \pm 0.06$ & $0.04 \pm 0.00$ & $186.9 \pm 14.6$ & $828 \pm 50$ \\
\hline & & Winter $2014 / 2015$ & $1633 \pm 358$ & $6.99 \pm 0.03$ & $0.06 \pm 0.01$ & $208.4 \pm 34.8$ & $896 \pm 116$ \\
\hline & & Early spring 2015 & $2021 \pm 238$ & $6.64 \pm 0.13$ & $0.03 \pm 0.00$ & $235.4 \pm 23.8$ & $988 \pm 78$ \\
\hline \multirow[t]{4}{*}{ C. rubrum } & \multirow[t]{4}{*}{ Cassis } & Summer 2014 & $1660 \pm 80$ & $2.32 \pm 0.18$ & $0.01 \pm 0.00$ & $98.3 \pm 2.9$ & $608 \pm 15$ \\
\hline & & Winter 2014/2015 & $1575 \pm 121$ & $2.68 \pm 0.10$ & $0.01 \pm 0.00$ & $94.6 \pm 7.0$ & $588 \pm 36$ \\
\hline & & Early spring 2015 & $1563 \pm 70$ & $2.86 \pm 0.08$ & $0.01 \pm 0.00$ & $92.2 \pm 4.0$ & $575 \pm 21$ \\
\hline & & Late spring 2015 & $1496 \pm 122$ & $2.62 \pm 0.16$ & $0.01 \pm 0.00$ & $89.4 \pm 3.8$ & $561 \pm 20$ \\
\hline Seawater & Cassis & Summer 2014 & $2931 \pm 468$ & $6.65 \pm 0.06$ & $0.01 \pm 0.00$ & $327.8 \pm 66.5$ & $1627 \pm 266$ \\
\hline & & Winter 2014/2015 & $2595 \pm 68$ & $6.98 \pm 0.08$ & $0.01 \pm 0.00$ & $335.6 \pm 19.3$ & $1664 \pm 77$ \\
\hline & & Early spring 2015 & $3629 \pm 5$ & $6.71 \pm 0.08$ & $0.01 \pm 0.00$ & $441.5 \pm 2.2$ & $2069 \pm 8$ \\
\hline & & Late spring 2015 & $3647 \pm 343$ & $6.67 \pm 0.07$ & $0.01 \pm 0.00$ & $430.2 \pm 25.1$ & $2026 \pm 93$ \\
\hline
\end{tabular}

(Suppl. File S3). Interestingly, the abundances of these three OTUs seemed to vary significantly among host species and appear to depend on sampling location (Fig. 4; Suppl. File S3). Each host species also harboured a few additional low abundant core microbes (e.g. Endozoicomonas OTU53279 and OTU75183 in E. cavolini and E. singularis; OTU79173 in L. sarmentosa) (Fig. 4; Suppl. File 3). We also analysed which OTUs were consistently present within one host species in at least one sampling location (locally stable microbial associates [7]) to identify bacteria that may contribute to local adaptation, and found that those were in fact commonly found at low abundances in most (but not all) samples from several species at both locations (Fig. 4; Suppl. File S3). Overall, this shows that there is significant overlap in the (core) microbiome (here set conservatively at an OTU presence in $100 \%$ of samples of a species) membership among 
Fig. 3 Overview of the composition of the bacterial community associated with Mediterranean octocorals at two locations on seasonal scales. Microbiome community composition is presented at the genus level (or lowest possible taxonomic level (p. phylum; o. order; f. family; g. genus)) for Corallium rubrum, Eunicella cavolini, E. singularis, E. verrucosa and Leptogorgia sarmentosa. The contribution of each taxon is indicated in percentages $(\%)$
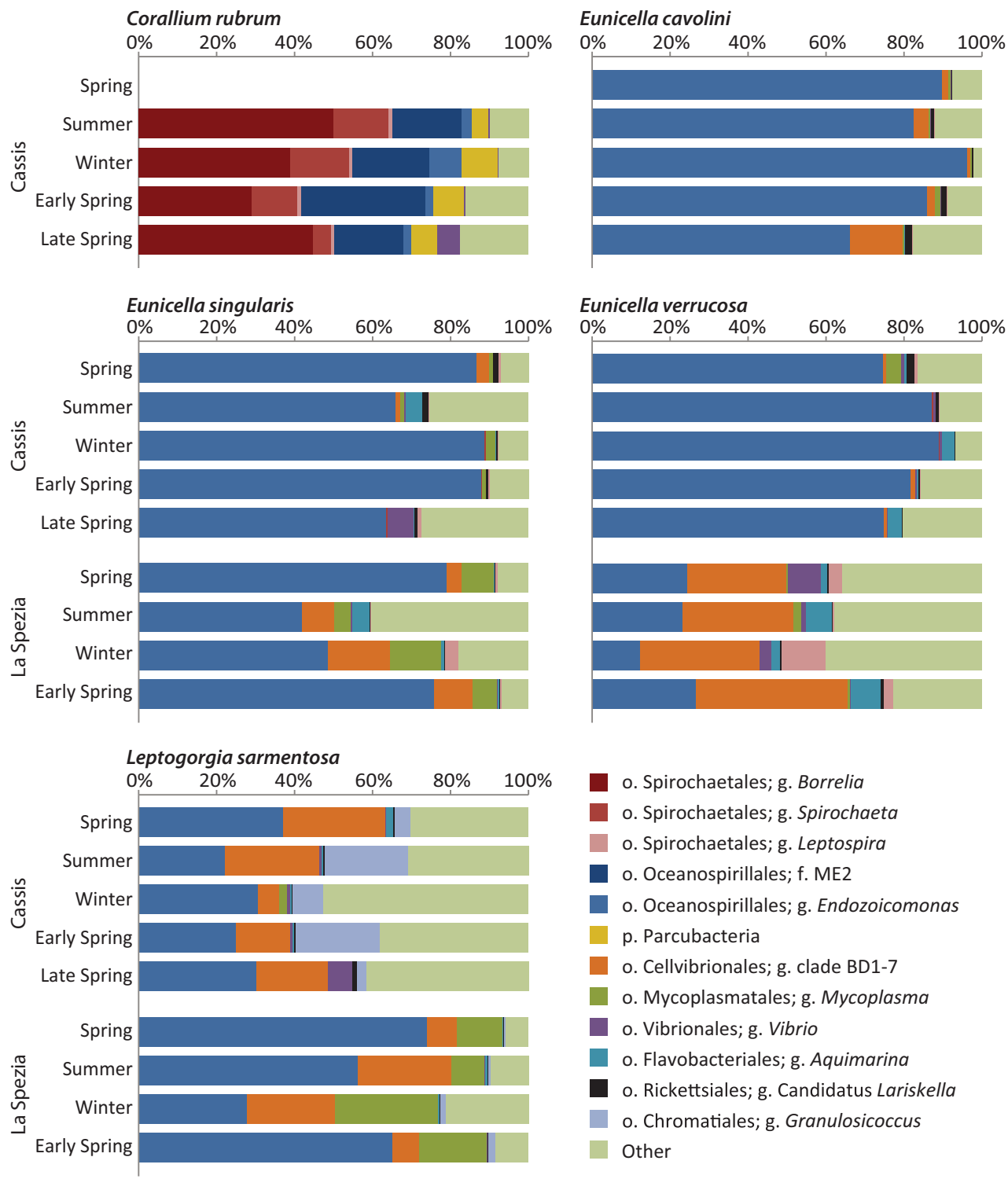

o. Spirochaetales; g. Borrelia

o. Spirochaetales; g. Spirochaeta

o. Spirochaetales; g. Leptospira

o. Oceanospirillales; f. ME2

o. Oceanospirillales; g. Endozoicomonas

p. Parcubacteria

o. Cellvibrionales; g. clade BD1-7

o. Mycoplasmatales; g. Mycoplasma

o. Vibrionales; g. Vibrio

o. Flavobacteriales; g. Aquimarina

o. Rickettsiales; g. Candidatus Lariskella

o. Chromatiales; g. Granulosicoccus

Other
Mediterranean gorgonians. Examples of locally stable microbial associates of gorgonians belonged to the genera Mycoplasma, Vibrio (incl. 100\% match to the coral pathogen V. shiloi) and Leptospira as well as some not clearly defined candidate genera (Lariskella, Hepatoplasma, Fritschea) and members of the Chromatiales-family Granulosicoccus and the order Flavobacterales (Fig. 3; Suppl. File S3).

\section{Temporal Changes in Gorgonian Microbiome Composition}

Despite its general dominance, the contributions of the core microbiomes changed over time in all gorgonian host species, but no consistent patterns were observed (Suppl. File S3). Specifically, the contributions of E. cavolini and E. singularis core microbiomes were reduced in summer and late spring in Cassis, but only in summer in La Spezia. In E. verrucosa, the changes were minor, although the core microbiome was more abundant in Cassis compared to La Spezia. The opposite was observed, however, in L. sarmentosa, which also showed reduced core microbiome contributions in winter. When also considering the locally stable microbial associates, we found that this particularly affected the microbiome of L. sarmentosa (Suppl. File S3).

At Cassis, in-depth analysis of temporal changes in the microbiomes using differential OTU abundance analysis, showed very few changes in the relative amount of specific OTUs in Eunicella spp. among time points (Suppl. File S4), which was consistent with the patterns in beta diversity. The most notable difference was the significant increase in the OTU corresponding to Vibrio shiloi (OTU64664) in the bacterial community of E. singularis in the late spring of 2015 compared to the other time points (Figs. 3 and 4; Suppl. File S4). A similar, but non-significant, trend in this OTU's presence was also observed in L. sarmentosa (Fig. 4). Comparisons of the L. sarmentosa 

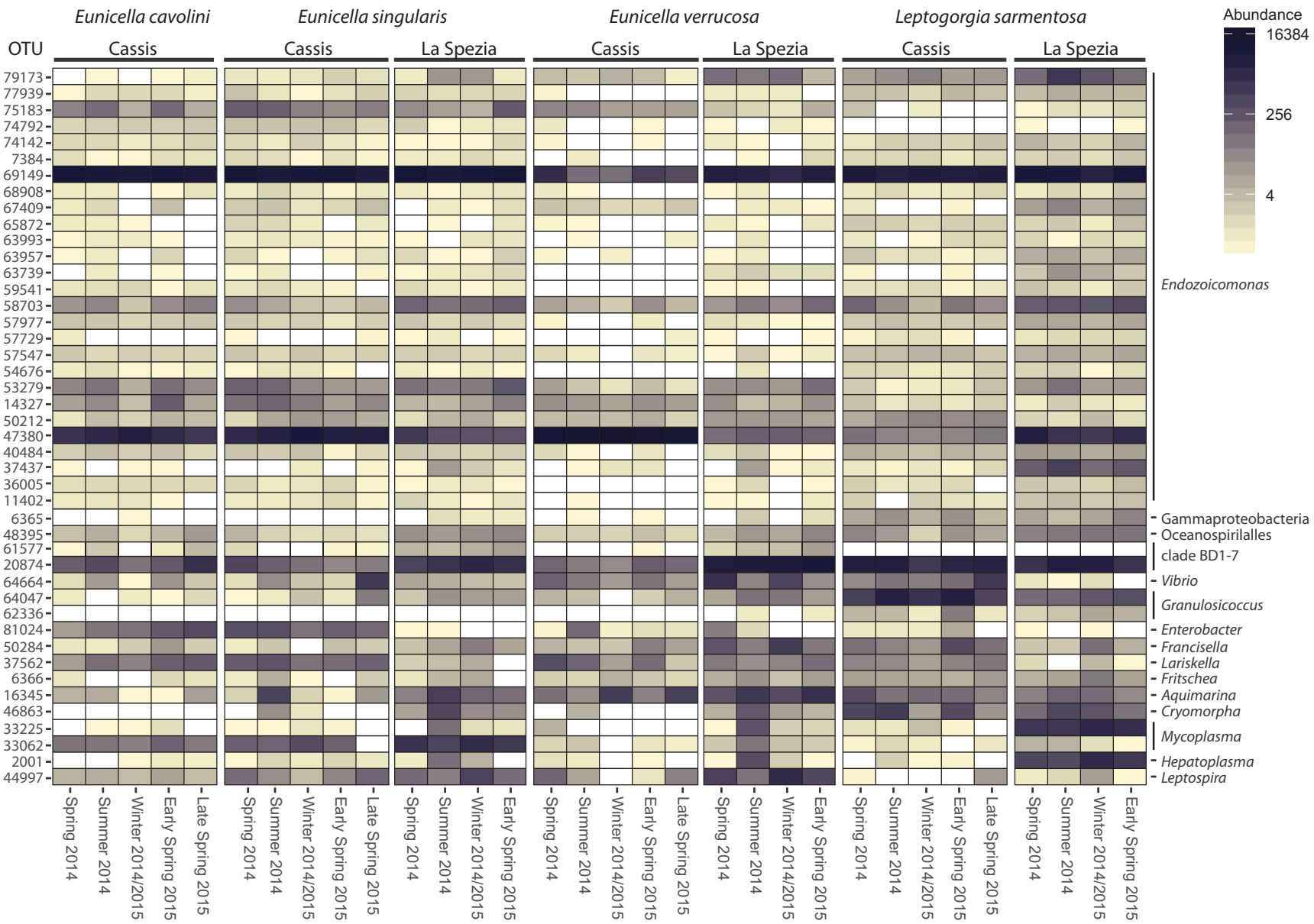

Fig. 4 Seasonal patterns in abundance of core and locally variant microbes of Mediterranean soft gorgonians. Heatmap presents the abundance of OTUs considered as part of the core microbiome and locally stable microbial associates of the gorgonians Eunicella cavolini,

E. singularis, E. verrucosa, Leptogorgia sarmentosa at two different locations - Cassis and La Spezia. The taxonomy of each OTU is given at the genus level (or lowest possible taxonomic level)

microbiome among all time points revealed some changes in the relative numbers of some generally low abundant OTUs, particularly between spring and winter, explaining the change in beta diversity observed. Between spring 2014 and winter 2014/2015, 62 OTUs were found to be differentially abundant, and 22 OTUs between the winter 2014/2015 and early spring 2015 (Suppl. File S4). The main patterns observed were (1) a decreased abundance of five OTUs commonly associated with gorgonians (Oceanospirillales (OTU48395), clade BD1-7 (OTU58703), Granulosicoccus (OTU62336), Francisella (OTU50284) and JTB225 (OTU21753) in winter and (2) an increase in OTUs that are related to the genera Caedibacter, Francisella and likely Coxiella in spring (Fig. 4; Suppl. File S4).

Although no seasonal changes in microbiome beta diversity was observed in Eunicella spp. in La Spezia, numerous OTUs were differentially abundant between seasons (Fig. 4; Suppl. File S4). Most notably, the abundances of common Mycoplasma and Hepatoplasma OTUs were higher in summer, despite an apparent decrease in Mycoplasma abundance (Fig. 3), while the common Leptospira (OTU44997) was reduced in abundance at this time. Similarly, we observed an increase in abundance of a Vibrio OTU (OTU49555) in summer, despite a reduction in the number of a common Vibrio OTU (OTU64664). In addition, the numbers of various Enterobacterales species were found to be significantly increased in spring compared with summer and winter. Despite the observed differences in beta diversity in the $L$. sarmentosa microbiome between spring on the one hand and summer and winter on the other hand at La Spezia, the number of differentially abundant OTUs was rather limited. In fact, the main drivers were the core Endozoicomonas and Cellvibrionales OTUs. Of particular interest was the lower abundance of Endozoicomonas OTU79173 and 'clade BD1-7' OTU20874 versus the increased numbers of Endozoicomonas OTU47380 and OTU69149 during spring time.

\section{Spatial Differences in Gorgonian Microbiome Composition}

Differential OTU abundance analysis showed that there were also major differences in the microbiomes of gorgonians 
between Cassis and La Spezia, corroborating the beta diversity results. However, the differences in the E. verrucosa-associated bacterial community (109 differentially abundant OTUs) could primarily be attributed to a 123 -fold reduced abundance of the generally most dominant Endozoicomonas in Mediterranean gorgonians (OTU47380) and an increase in the relative numbers of other common microbial associates, such as 'clade BD1-7' (OTU20874), Endozoicomonas (OTU69149), Leptospira (OTU44997) and Aquimarina (OTU16345) as well as numerous low abundant OTUs, including various other Endozoicomonas and Cellvibrionales OTUs plus multiple Legionellales and Enterobacterales (Fig. 4; Suppl. Files 3 and 4). Similar spatial differences were observed in the microbiome of E. singularis (42 differentially abundant OTUs), with the particular notion that there was a major increase in the abundance of Mycoplasma (OTU33062) at La Spezia compared with Cassis (Fig. 4; Suppl. Files 3 and 4). L. sarmentosa (528 differentially abundant OTUs) also showed a high abundance of the two Mollicutes genera Mycoplasma, although these were different OTUs (most dominant OTU23384 and OTU33225), and Hepatoplasma (OTU2001) at La Spezia (Fig. 4; Suppl. Files 3 and 4). In contrast to Eunicella spp., the microbiome of L. sarmentosa contained relatively higher numbers of the main Endozoicomonas OTUs (OTU47380 and OTU69149) at La Spezia, while OTUs identified as Vibrio and Chromatiales were significantly more abundant in Cassis (Fig. 4; Suppl. Files 3 and 4).

\section{Composition and Temporal Patterns of the Microbiome of the Red Coral Corallium rubrum}

The diversity of the microbiome of C. rubrum was significantly different from seawater bacterial communities (1B, Table 2, Suppl. File S1-Table S1) at all time points (Suppl. File S1Table S2). However, no significant changes in alpha and beta diversity were observed in the red coral's microbiome on a temporal scale (Fig. 2; Suppl. File S1-Table S4). Members of the classes Spirochaetes (order Spirochaetales) and Gammaproteobacteria (order Oceanospirillales) and the phylum Parcubacteria dominated the bacterial assemblages of C. rubrum (Fig. 3; Suppl. File S2). In-depth analysis revealed that 44 OTUs made up the core microbiome, of which only 7 represented 86$95 \%$ of the overall bacterial community (Suppl. File S3). These 7 OTUs were 1 Parcubacteria OTU, 3 Spirochaetales OTUs (1 Leptospiraceae, 2 Spirochaetaceae) and 3 Oceanospirillales (1 Endozoicomonas, $1 \mathrm{ME2}$ and 1 unclassified). All other core OTUs were very low abundant $(<1 \%)$, but included 6 Endozoicomonas OTUs, 7 ME2 OTUs and 3 Spirochaetaceae OTUs as well as 1 Cellvibrionales BD1-7 OTU and 2 Propionibacterium OTUs (Suppl. File S3).

While no temporal changes in overall microbiome diversity were observed, few OTUs showed changes in abundance between seasons (Fig. 5; Suppl. File S4); particularly several Pseudoalteromonadaceae OTUs were more abundant during summer. Core microbes were generally stable in abundance over time, for example no Parcubacteria and Spirochaetales core OTU was found differentially abundant between seasons. However, some core Gammaproteobacteria did change in relative abundance, including the most abundant Endozoicomonas (OTU 30320), which was more abundant in winter compared to early spring, and the most abundant ME2 (OTU20697), which was more abundant in early spring compared to summer, and could explain the difference in ME2 and Endozoicomonas abundances observed at these time points (Figs. 3 and 5). In addition, a low abundant unclassified Gammaproteobacteria (OTU 4533) was generally found at higher abundance in spring than in winter or summer. All other OTUs found differentially abundant between seasons were primarily very low abundant Gammaproteobacteria that are not part of the core microbiome.

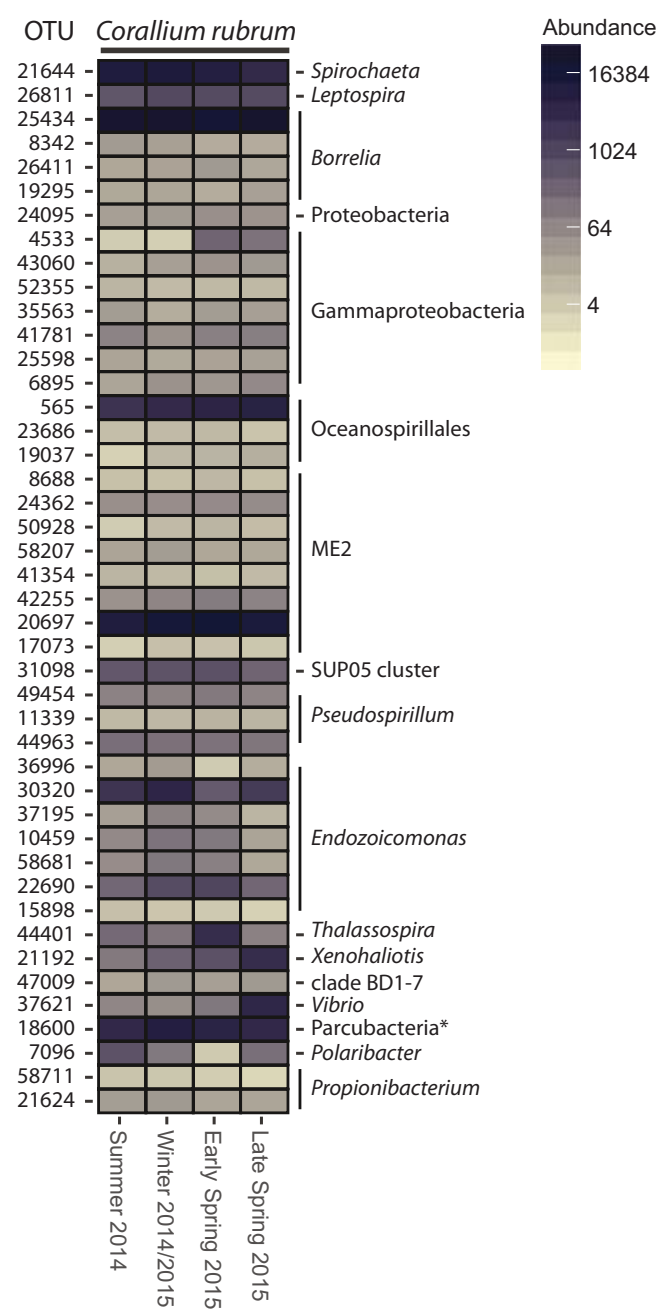

Fig. 5 Seasonal patterns in abundance of core microbes of the red coral Corallium rubrum. Heatmap presents the standardized abundance of core OTUs in the bacterial community associated with C. rubrum at Cassis on seasonal scales. The taxonomy of each OTU is given at the genus level (or lowest possible taxonomic level) 


\section{Discussion}

In this study, we show that the microbiomes of five Mediterranean octocorals are highly structured, being dominated by their core microbes, and are relatively stable over time. The microbiomes of all soft gorgonian species showed significant overlap in bacterial associates, and were composed primarily of the same Endozoicomonas and Cellvibrionales BD1-7 bacteria. The red coral microbiome was dominated by Spirochaetales, the Oceanospirillales family ME2 and a member of the Parcubacteria, while Endozoicomonas played a relatively minor role. The temporal dynamics in the abundances of the gorgonian-associated bacteria appeared to largely depend on the location they lived at; however, it is unclear whether these are adverse impacts on holobiont physiology or adaptation to local conditions.

\section{Core and Locally Stable Bacteria in the Microbiome of Mediterranean Soft Gorgonians}

Consistent with our previous study on the spatial stability of the microbiomes of Mediterranean soft gorgonians [7], we show that the bacterial communities of Eunicella spp. and Leptogorgia sarmentosa are highly dominated by only a few bacterial species, particularly from the Oceanospirillales genus Endozoicomonas and the Cellvibrionales clade BD1-7. The order Cellvibrionales was proposed only relatively recent [47], and Alteromonadales clade BD1-7 bacteria found as core microbes in Eunicella cavolini [7] have now been assigned to this new order. In our current study, using higher sequencing depth than before, we actually found that this bacterium is consistently associated with all gorgonians of the Gorgoniidae family in the Mediterranean Sea, suggesting that it is crucial for the health of these holobionts. Although the function of these bacteria is currently still unknown, genetic studies have suggested that BD1-7 bacteria are oligotrophs and may have the potential to use alternative energy sources for mixotrophic growth, such as light for the generation of ATP via proteorhodopsin proton pump [48, 49].

The most dominant bacterial associates of all gorgonians from the Gorgoniidae family belonged to the genus Endozoicomonas. In fact, all species assessed in this study possessed the same main Endozoicomonas OTUs in their bacterial community, showing their particular importance for gorgonian holobiont functioning. These bacteria are often found in the microbiome of corals, gorgonians and other marine invertebrates, and are considered crucial for host health [23, $33,50-54]$, via their involvement in microbiome structuring, through secretion of antibiotic and quorum-sensing compounds [3, 55-57], and nutrient cycling and acquisition [10, $20,55,58-61]$. The relative stability of the association and abundance of each OTU show that local environmental changes had relatively little impact and suggest that they may be protectively harboured within the tissues of the host, as found in tropical corals and anemones [3, 62]. Whether the Endozoicomonas species found in our study are indeed present in large aggregates in the host tissues and which functions they perform, is currently under investigation.

Interestingly, we also found that one OTU matching the coral pathogen Vibrio shiloi was commonly present on the gorgonians. Vibrio species have previously been implicated in disease in both tropical and Mediterranean scleractinians and gorgonians. For example, strains of $V$. coralliilyticus have been identified as the causative agent of disease in the Mediterranean gorgonian Paramuricea clavata [34, 35] and in tropical corals [63, 64], while $V$. shiloi has been implicated in bacteria-induced bleaching in Oculina patagonica [65]. Recently, it was reported that $V$. shiloi was only found in disease lesions and is generally not associated with $O$. patagonica, and together with V. coralliilyticus synergistically causes disease [66]. However, we found $V$. shiloi to be consistently present with all gorgonians year-round at low abundances, although in the late spring of 2015 its abundance showed an increasing trend. As all gorgonians were visually healthy at the time of sampling, this suggests that this bacterium may be an opportunistic pathogen and only cause disease when the environmental conditions increase its virulence and the integrity of the holobiont is compromised.

Another interesting association was found between all Gorgoniidae species and OTUs of the Mollicutes genera Mycoplasma and Hepatoplasma; however, their abundance was particularly high in L. sarmentosa and E. singularis in La Spezia. Interestingly, the most abundant Mycoplasma OTU in L. sarmentosa was different from the one found at high abundance in E. singularis, suggesting host preference. While Mycoplasma are generally considered to be intracellular parasites of plants and animals, species associated with gorgonians [67-69] and the coldwater coral Lophelia pertusa [70, 71] have been suggested to be commensals or symbionts. In L. pertusa, for example, the Mycoplasma bacteria were found extracellularly adjacent to the coral's spirocysts, which lead to the hypothesis that they benefit from hemolymph components leaking from prey captured by the host, without affecting host health [71]. Hepatoplasma bacteria were relatively rare in Cassis, but more common at La Spezia, particularly in association with L. sarmentosa. Sequences related to Hepatoplasma have previously been found in various cnidarians, including Nematostella vectensis [72] and gorgonians [67]. Although the origin and function of these bacteria are unknown, extensive studies on Hepatoplasma in terrestrial isopods suggest they are ectosymbionts [73, 74]. While Hepatoplasma could thus be a symbiont of anthozoans aiding in nutrient metabolism of the holobiont, it cannot be excluded that this bacterium originates from the planktonic arthropods that gorgonians prey on.

We also observed that the gorgonians commonly interacted with Granulosicoccus, Lariskella, and Fritschea bacteria. While currently nothing is known about the role of these bacteria in cnidarian biology, they have previously been found in these 
animals. For example, our Candidatus Lariskella OTU shared $100 \%$ homology with a Rickettsiales bacterium found in the freshwater Hydra [75], and members of this candidate genus have generally be considered endosymbionts in arthropods hosts [76]. The Chlamydiae Candidatus Fritschea has been discovered in tropical corals [50,77], a jellyfish (Viver et al. unpublished) and other marine invertebrates [78, 79]. Members of the family Granulosicoccus (order Chromatiales) are often found associated with algae and seagrass, but $16 \mathrm{~S}$ sequences matching 99 and $100 \%$ with the common Granulosicoccus OTUs in our study have recently been detected in Eunicella cavolini [21] and various tropical scleractinian corals $[50,77]$, respectively.

\section{Temporal and Spatial Stability of the Gorgonian Microbiome}

While the gorgonian-associated microbiomes were generally composed of the same core microbial species and locally stable microbial associates, our main objective was to investigate the stability of these associations over time. Seasonal differences in bacterial communities have been reported for several scleractinian and gorgonian coral species at different geographical scales [80-83]. Although observed in the Mediterranean cold-water coral Madrepora oculata [84] and the gorgonian P. clavata [85], the temporally stable interactions between Mediterranean gorgonians and their microbial associates are remarkable, particularly because of major differences in seawater temperature and rainfall between the sampling months (Table 3), and suggest tight microbiome regulation by these host animals. Even on the OTU level, relatively few changes were observed, which mostly involved low abundant bacteria that did not belong to the core or common microbiome. The only exception was $L$. sarmentosa at La Spezia in which the microbiome underwent significant modifications in composition over time, but which were primarily driven by a re-shuffling in the abundances of the main core microbes and the locally stable microbial associates. Overall, this indicates that the L. sarmentosa holobiont may be more flexible and able to adapt to the environmental conditions. Significant shifts in the bacterial communities of the gorgonians and at the OTU level mostly took place between spring time points on the one hand and summer and winter time points on the other. As environmental conditions rapidly change during spring, this may be the most likely factor responsible for the observed microbiome shifts.

Although the gorgonian-associated microbiomes were generally stable over time, we did observe major differences between the two sampling sites. The Cassis and La Spezia locations are characterized by very different environmental conditions: The water at the Cassis location is relatively clear and unaffected by terrestrial input, while the La Spezia location is impacted by river and sewage influxes resulting in high levels of turbidity and pollution. These dissimilarities in the local environment may explain the differences observed here in the gorgonian-associated bacterial communities. Recently, we reported that the gorgonian microbiome is relatively stable on spatial scales, but that local disturbances may indeed impact the bacterial community associated with these animals [7]. In this study, however, our results indicate that the extent of the impacts on the microbiome appears to be highly species-dependent, but also raise questions as to what may constitute a 'healthy' gorgonian microbiome. For example, the abundance of core and common microbiome members was significantly lower in E. verrucosa near La Spezia compared with Cassis; particularly the most abundant Endozoicomonas OTU47380. Reduction in Endozoicomonas abundance in corals is often characteristic of stress $[51,52,55,77,86]$ and combined with the increased diversity observed suggests that the $E$. verrucosa microbiome was negatively impacted at this turbid location. Surprisingly, however, the bacterial community associated with $L$. sarmentosa showed the opposite pattern, being significantly more dominated by core and common bacterial associates at La Spezia, which indicates that this holobiont may in fact have been healthier under the same conditions. These contrasting signatures in the bacterial communities of these two gorgonians from the Gorgoniidae family suggest that these species may still have different habitat preferences despite living sympatrically close to muddy bottoms [87]. As some core bacteria, such as Endozoicomonas OTU47380, were reduced in abundance in E. verrucosa, but showed higher abundance in L. sarmentosa at La Spezia, the environmental conditions probably did not affect the viability of these microbes and provides further support for host control of the microbiome. Which factors are responsible for these differences in bacterial assemblages, however, remains to be investigated. Overall, our data shows that the microbiome of
Table 3 Environmental conditions at the sampling locations

\begin{tabular}{|c|c|c|c|c|c|c|}
\hline \multirow[b]{2}{*}{ Location } & \multirow[b]{2}{*}{ Species/seawater } & \multicolumn{3}{|l|}{2014} & \multicolumn{2}{|l|}{2015} \\
\hline & & Spring & Summer & Winter & Early spring & Late spring \\
\hline \multirow[t]{2}{*}{ Cassis } & Seawater temperature $\left({ }^{\circ} \mathrm{C}\right)$ & 15.9 & 21.3 & 17.5 & 13.9 & 17.8 \\
\hline & Rainfall (mm) & 32.1 & 90.8 & 191.6 & 62.0 & 90.6 \\
\hline \multirow[t]{2}{*}{ La Spezia } & Seawater temperature $\left({ }^{\circ} \mathrm{C}\right)$ & 15.9 & 22.7 & 13.8 & 14.1 & \\
\hline & Rainfall (mm) & 32.0 & 41.1 & 94.2 & 43.2 & \\
\hline
\end{tabular}


the Mediterranean soft gorgonians is relatively stable over time and under control of the host, but that local conditions shape its associated bacterial communities.

\section{Microbiome of the Red Coral Corallium rubrum}

The microbiome of the red coral was highly distinct from any known cnidarian-associated bacterial community, including the Mediterranean soft gorgonians. Spirochaetales (genera Borrelia, Spirochaeta and Leptospira) were the most dominant bacteria, followed by the Oceanospirillales (ME2 family, an unknown Oceanospirillales and Endozoicomonas) and an OTU belonging to the relatively unknown phylum Parcubacteria (previously classified as Candidate Division OD1) [18]. The core microbiome consisted of 7 main OTUs, representing $86.4-94.5 \%$ of the microbiome, in addition to 37 low abundant $(<1 \%)$ OTUs. This highly structured microbiome of $C$. rubrum is consistent with our study on the spatial stability of the red coral microbiome throughout the Mediterranean Sea [18] and suggests that the microbiome is not only stable on spatial scales, but also over time ( $>2$ years). Indeed, we did not find any significant temporal changes within the red coral-associated bacterial community in this study. Overall, our results indicate that the red coral holobiont possesses strong bacterial selection mechanisms to maintain a healthy microbiome. Octocorals are known to produce secondary metabolites with antimicrobial activity which may be used by the host to regulate its microbiome [29-32]; however, members of the coralassociated microbiome may also competitively prevent nonsymbiotic residents from entering the bacterial community through nutrient competition and antibiotic production.

While the exact function of the bacteria within the C. rubrum microbiome is still unknown, the consistent association of the main OTUs on temporal and spatial scales provides strong evidence for symbiotic relationships. Spirochaetes were found to be relatively dominant bacteria associated with some octocorals only recently $[18,25,26]$, although they have been observed at low abundance on scleractinian corals before [4, 70, 88, 89]. While the order Spirochaetales harbours multiple pathogens, some species are known endosymbionts [90-92] involved in digestion as well as carbon and nitrogen fixation into bioavailable nutrients for the hosts $[27,28]$. The Spirochaetales associated with the red coral may provide similar functions to the holobiont as previously suggested and may also be implicated in regulation of the microbiome [18]. The Parcubacteria are largely unknown bacteria that have primarily been found living in anoxic conditions [93], and recent genomic investigations have shown that they have severely reduced metabolic capabilities [94]. For example, they are incapable of producing cofactors, nucleotides, amino acids and fatty acids. This lack of biosynthesis machinery suggests a highly specialised life-style, likely of symbiotic nature [94]. It remains, however, unclear what services Parcubacteria provide to their host. The other main group of bacteria associated with the red coral are members of the order Oceanospirillales. Endozoicomonas are probably the best studied coral-associated bacteria, but appear to play only minor roles in the C. rubrum holobiont. Members of the relatively unknown ME2 family may, however, fulfil the same function in the red coral as the Endozoicomonas in other corals whose microbiome is dominated by bacteria from this genus.

\section{Co-evolution of the Gorgonian-Associated Microbiome}

To explain the regulation of microbiome composition within the holobiont, two theories have been postulated: the holobiont model [15] and the hologenome theory of evolution [95]. Although both theories state that the host shapes its microbiome based on metabolism and the use of antimicrobials but that local conditions can impact its membership, we previously argued that the holobiont model applies better to the Mediterranean gorgonian holobionts [7]. Our main argument was that the hologenome theory of evolution includes heredity (i.e. transmission of bacteria between generations), which requires that (1) these symbionts are ubiquitous and (2) there are strong phylosymbiotic signals, but that such phylosymbiotic signals were absent in the gorgonian-associated microbiomes due to the significant overlap in ubiquitous bacteria (core and common microbial associates; which are in at least all gorgonians from the Gorgoniidae family mostly the same). Taken together, our studies (here and $[7,18]$ ) covering six octocoral species from two taxonomic sub-orders, three families and four genera (Fig. 6) show that there is significant divergence in microbiome composition along host phylogenetic lines. However, these co-evolutionary signals appear to end at the family level as gorgonian species from different genera within the same family show significant overlap in microbiome composition at the bacterial species level (97\% identity similarity). As such, these ancient host-microbe associations have been highly conserved through evolutionary time, but differences between the microbiomes of the Gorgoniidae species may still exist at the bacterial strain level. Further investigations at the strain level of the various ubiquitous microbes could provide us with a higher resolution on phylosymbiotic signals in the gorgonian holobionts and may show strain specificity among the various

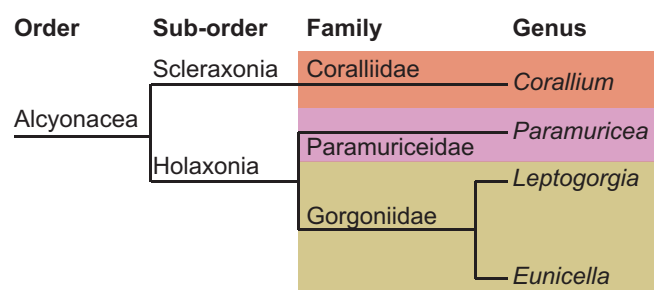

Fig. 6 Schematic overview of the taxonomy of the Mediterranean octocorals whose microbiome was analysed here and in our previous studies [7, 18]. The different colours identify taxa harbouring distinct core microbiomes. Taxa grouped by colour, e.g. genera within the Gorgoniidae, show significant overlap in (core) microbiome composition 
gorgonian holobionts. However, our current data provides additional support for the holobiont model.

\section{Concluding Remarks}

In this study, we provide an in-depth analysis of the bacterial community of five Mediterranean gorgonians from two different taxonomic sub-orders on temporal scales. Overall, we show that their highly structured microbiomes are relatively stable over time, but are likely adjusted to local environmental conditions, supported by the holobiont model. The strong and specific associations of the host with up to 7 dominant bacterial species and $\sim 40$ common low abundant associates show tight regulation of the holobiont membership. Divergence in microbiome composition was, however, clear along distant phylogenetic lines, although there is significant overlap among species belonging to the same family. The physiological contributions of these microbial symbionts to host health remain to be elucidated.

Acknowledgements The authors would like to thank Stéphanie Reynaud and Eric Beraud from the Centre Scientifique de Monaco, and Gabriella Cerrati and Andrea Bordone from ENEA for help with sample collections. Craig Michell is thanked for his assistance with the 16S gene amplicon library preparation. We would also like to thank the Paul Hamel Foundation and KAUST for financial support.

\section{Compliance with Ethical Standards}

Conflict of Interest The authors declare that they have no conflict of interest.

\section{References}

1. Bosch TCG, McFall-Ngai MJ (2011) Metaorganisms as the new frontier Zoology (Jena, Germany) 114(4):185-190

2. McFall-Ngai M, Hadfield MG, Bosch TCG, Carey HV, DomazetLošo T, Douglas AE, Dubilier N, Eberl G, Fukami T, Gilbert SF, Hentschel U, King N, Kjelleberg S, Knoll AH, Kremer N, Mazmanian SK, Metcalf JL, Nealson K, Pierce NE, Rawls JF, Reid A, Ruby EG, Rumpho M, Sanders JG, Tautz D, Wernegreen JJ (2013) Animals in a bacterial world, a new imperative for the life sciences Proc Natl Acad Sci 110(9):3229-3236

3. Bayer T, Neave MJ, Alsheikh-Hussain A, Aranda M, Yum LK, Mincer T, Hughen K, Apprill A, Voolstra CR (2013) The microbiome of the Red Sea coral Stylophora pistillata is dominated by tissue-associated Endozoicomonas bacteria Appl Environ Microbiol 79(15):4759-4762

4. Ainsworth TD, Krause L, Bridge T, Torda G, Raina J-B, Zakrzewski M, Gates RD, Padilla-Gamino JL, Spalding HL, Smith C, Woolsey ES, Bourne DG, Bongaerts P, HoeghGuldberg O, Leggat W (2015) The coral core microbiome identifies rare bacterial taxa as ubiquitous endosymbionts Isme J 9(10): 2261-2274

5. Hernandez-Agreda A, Gates RD, Ainsworth TD Defining the core microbiome in microbial soup Trends Microbiol 25(2):125-140
6. Hernandez-Agreda A, Leggat W, Bongaerts P, Ainsworth TD (2016) The microbial signature provides insight into the mechanistic basis of coral success across reef habitats mBio 7 (4)

7. van de Water JAJM, Melkonian R, Voolstra CR, Junca H, Beraud E, Allemand D, Ferrier-Pagès C (2016) Comparative assessment of Mediterranean gorgonian-associated microbial communities reveals conserved core and locally variant bacteria Microb Ecol 73(2):466-478

8. Bednarz VN, Grover R, Maguer J-F, Fine M, Ferrier-Pagès C (2017) The assimilation of diazotroph-derived nitrogen by scleractinian corals depends on their metabolic status MBio 8(1): e02058-16

9. Lema KA, Willis BL, Bourne DG (2012) Corals form characteristic associations with symbiotic nitrogen-fixing bacteria Appl Environ Microbiol 78(9):3136-3144

10. Raina JB, Tapiolas D, Willis BL, Bourne DG (2009) Coralassociated bacteria and their role in the biogeochemical cycling of sulfur Appl Environ Microbiol 75(11):3492-3501

11. Kvennefors EC, Sampayo E, Kerr C, Vieira G, Roff G, Barnes AC (2012) Regulation of bacterial communities through antimicrobial activity by the coral holobiont Microb Ecol 63(3):605-618

12. Nissimov J, Rosenberg E, Munn CB (2009) Antimicrobial properties of resident coral mucus bacteria of Oculina patagonica FEMS Microbiol Lett 292(2):210-215

13. Ritchie KB (2006) Regulation of microbial populations by coral surface mucus and mucus-associated bacteria Mar Ecol Prog Ser 322:1-14

14. Shnit-Orland M, Sivan A, Kushmaro A (2012) Antibacterial activity of Pseudoalteromonas in the coral holobiont Microb Ecol 64(4): 851-859

15. Rohwer F, Seguritan V, Azam F, Knowlton N (2002) Diversity and distribution of coral-associated bacteria Mar Ecol Prog Ser 243:1-10

16. Rypien KL, Ward JR, Azam F (2010) Antagonistic interactions among coral-associated bacteria Environ Microbiol 12(1):28-39

17. van de Water JAJM, Lamb JB, van Oppen MJH, Willis BL, Bourne DG (2015) Comparative immune responses of corals to stressors associated with offshore reef-based tourist platforms Conserv Physiol 3(1): cov032

18. van de Water JAJM, Melkonian R, Junca H, Voolstra CR, Reynaud S, Allemand D, Ferrier-Pagès C (2016) Spirochaetes dominate the microbial community associated with the red coral Corallium rubrum on a broad geographic scale Sci Rep 6:27277

19. Ballesteros E (2006) Mediterranean coralligenous assemblages. In: Oceanography and Marine Biology. Oceanography and Marine Biology-An Annual Review. CRC Press, pp 123-195.

20. Correa H, Haltli B, Duque C, Kerr R (2013) Bacterial communities of the gorgonian Octocoral Pseudopterogorgia elisabethae Microb Ecol 66(4):972-985

21. Bayer T, Arif C, Ferrier-Pagès C, Zoccola D, Aranda M, Voolstra C (2013) Bacteria of the genus Endozoicomonas dominate the microbiome of the Mediterranean gorgonian coral Eunicella cavolini Mar Ecol Prog Ser 479:75-84

22. La Rivière M, Garrabou J, Bally M (2015) Evidence for host specificity among dominant bacterial symbionts in temperate gorgonian corals Coral Reefs 34(4):1087-1098

23. Ransome E, Rowley SJ, Thomas S, Tait K, Munn CB (2014) Disturbance to conserved bacterial communities in the cold-water gorgonian coral Eunicella verrucosa FEMS Microbiol Ecol 90(2): 404-416

24. Neave MJ, Apprill A, Ferrier-Pagès C, Voolstra CR (2016) Diversity and function of prevalent symbiotic marine bacteria in the genus Endozoicomonas Appl Microbiol Biotechnol 100(19): 8315-8324

25. Wessels W, Sprungala S, Watson SA, Miller DJ, Bourne DG (2017) The microbiome of the octocoral Lobophytum pauciflorum: minor 
differences between sexes and resilience to short-term stress FEMS Microbiol Ecol 93(5):fix013

26. Lawler SN, Kellogg CA, France SC, Clostio RW, Brooke SD, Ross SW (2016) Coral-associated bacterial diversity is conserved across two deep-sea Anthothela species Front Microbiol 7:458

27. Leadbetter JR, Schmidt TM, Graber JR, Breznak JA (1999) Acetogenesis from $\mathrm{H} 2$ plus $\mathrm{CO} 2$ by spirochetes from termite guts Science 283(5402):686-689

28. Lilburn TG, Kim KS, Ostrom NE, Byzek KR, Leadbetter JR, Breznak JA (2001) Nitrogen fixation by symbiotic and free-living spirochetes Science 292(5526):2495-2498

29. Jensen PR, Harvell CD, Wirtz K, Fenical W (1996) Antimicrobial activity of extracts of Caribbean gorgonian corals Mar Biol 125(2): 411-419

30. Kim K, Harvell CD, Kim PD, Smith GW, Merkel SM (2000) Fungal disease resistance of Caribbean sea fan corals (Gorgonia spp.) Mar Biol 136(2):259-267

31. Kim K (1994) Antimicrobial activity in gorgonian corals (Coelenterata, Octocorallia) Coral Reefs 13(2):75-80

32. Hunt LR, Smith SM, Downum KR, Mydlarz LD (2012) Microbial regulation in gorgonian corals Mar Drugs 10(6):1225-1243

33. Vezzulli L, Pezzati E, Huete-Stauffer C, Pruzzo C, Cerrano C (2013) 16SrDNA pyrosequencing of the Mediterranean gorgonian Paramuricea clavata reveals a link among alterations in bacterial holobiont members, anthropogenic influence and disease outbreaks PLoS One 8(6):e67745

34. Bally M, Garrabou J (2007) Thermodependent bacterial pathogens and mass mortalities in temperate benthic communities: a new case of emerging disease linked to climate change Glob Chang Biol 13(10):2078-2088

35. Vezzulli L, Previati M, Pruzzo C, Marchese A, Bourne DG, Cerrano C, the VibrioSea Consortium (2010) Vibrio infections triggering mass mortality events in a warming Mediterranean Sea Environ Microbiol 12(7):2007-2019

36. Coma R, Ribes M, Serrano E, Jiménez E, Salat J, Pascual J (2009) Global warming-enhanced stratification and mass mortality events in the Mediterranean Proc Natl Acad Sci 106(15):6176-6181

37. Cupido R, Cocito S, Barsanti M, Sgorbini S, Peirano A, Santangelo $G$ (2009) Unexpected long-term population dynamics in a canopyforming gorgonian coral following mass mortality Mar Ecol Prog Ser 394:195-200

38. Astraldi M, Gasparini G (1986) La circolazione costiera nel Mar Ligure Orientale Bollettino dei Musei e degli Istituti Biologici dell'Universita` di Genova 52:317-331

39. Andersson AF, Lindberg M, Jakobsson H, Bäckhed F, Nyrén P, Engstrand L (2008) Comparative analysis of human gut microbiota by barcoded pyrosequencing PLoS One 3(7):e2836

40. Camarinha-Silva A, Jáuregui R, Chaves-Moreno D, Oxley APA, Schaumburg F, Becker K, Wos-Oxley ML, Pieper DH (2014) Comparing the anterior nare bacterial community of two discrete human populations using Illumina amplicon sequencing Environ Microbiol 16(9):2939-2952

41. Caporaso JG, Kuczynski J, Stombaugh J, Bittinger K, Bushman FD, Costello EK, Fierer N, Pena AG, Goodrich JK, Gordon JI, Huttley GA, Kelley ST, Knights D, Koenig JE, Ley RE, Lozupone CA, McDonald D, Muegge BD, Pirrung M, Reeder J, Sevinsky JR, Turnbaugh PJ, Walters WA, Widmann J, Yatsunenko T, Zaneveld J, Knight R (2010) QIIME allows analysis of highthroughput community sequencing data Nat Methods 7(5):335-336

42. Quast C, Pruesse E, Yilmaz P, Gerken J, Schweer T, Yarza P, Peplies J, Glöckner FO (2013) The SILVA ribosomal RNA gene database project: improved data processing and web-based tools Nucleic Acids Res 41(D1):D590-D596

43. Edgar RC, Haas BJ, Clemente JC, Quince C, Knight R (2011) UCHIME improves sensitivity and speed of chimera detection Bioinformatics 27(16):2194-2200
44. Edgar RC (2010) Search and clustering orders of magnitude faster than BLAST Bioinformatics 26(19):2460-2461

45. McMurdie PJ, Holmes S (2013) phyloseq: an R package for reproducible interactive analysis and graphics of microbiome census data PLoS One 8(4):e61217

46. Love MI, Huber W, Anders S (2014) Moderated estimation of fold change and dispersion for RNA-seq data with DESeq2 Genome Biol 15(12):550

47. Spring S, Scheuner C, Goker M, Klenk HP (2015) A taxonomic framework for emerging groups of ecologically important marine gammaproteobacteria based on the reconstruction of evolutionary relationships using genome-scale data Front Microbiol 6:281

48. Spring S, Riedel T (2013) Mixotrophic growth of bacteriochlorophyll a-containing members of the OM60/NOR5 clade of marine gammaproteobacteria is carbon-starvation independent and correlates with the type of carbon source and oxygen availability BMC Microbiol 13(1):117

49. Oh H-M, Kang I, Ferriera S, Giovannoni SJ, Cho J-C (2010) Genome sequence of the oligotrophic marine Gammaproteobacterium HTCC2143, isolated from the Oregon Coast J Bacteriol 192(17): 4530-4531

50. Ziegler M, Roik A, Porter A, Zubier K, Mudarris MS, Ormond R, Voolstra CR (2015) Coral microbial community dynamics in response to anthropogenic impacts near a major city in the central Red Sea Mar Pollut Bull 105(2):629-640

51. Roder C, Bayer T, Aranda M, Kruse M, Voolstra CR (2015) Microbiome structure of the fungid coral Ctenactis echinata aligns with environmental differences Mol Ecol 24(13):3501-3511

52. Meyer JL, Paul VJ, Teplitski M (2014) Community shifts in the surface microbiomes of the coral Porites astreoides with unusual lesions PLoS One 9(6):e100316

53. Webster NS, Negri AP, Botté ES, Laffy PW, Flores F, Noonan S, Schmidt C, Uthicke S (2016) Host-associated coral reef microbes respond to the cumulative pressures of ocean warming and ocean acidification Sci Rep 6:19324

54. Gardères J, Bedoux G, Koutsouveli V, Crequer S, Desriac F, Pennec G (2015) Lipopolysaccharides from commensal and opportunistic bacteria: characterization and response of the immune system of the host sponge Suberites domuncula Mar Drugs 13(8):4985

55. Morrow KM, Bourne DG, Humphrey C, Botte ES, Laffy P, Zaneveld J, Uthicke S, Fabricius KE, Webster NS (2015) Natural volcanic $\mathrm{CO} 2$ seeps reveal future trajectories for host-microbial associations in corals and sponges Isme J 9(4):894-908

56. Jessen C, Villa Lizcano JF, Bayer T, Roder C, Aranda M, Wild C, Voolstra CR (2013) In-situ effects of eutrophication and overfishing on physiology and bacterial diversity of the Red Sea coral Acropora hemprichii PLoS One 8(4):e62091

57. Rua CPJ, Trindade-Silva AE, Appolinario LR, Venas TM, Garcia GD, Carvalho LS, Lima A, Kruger R, Pereira RC, Berlinck RGS, Valle RAB, Thompson CC, Thompson F (2014) Diversity and antimicrobial potential of culturable heterotrophic bacteria associated with the endemic marine sponge Arenosclera brasiliensis PeerJ 2: e419

58. Bourne DG, Dennis PG, Uthicke S, Soo RM, Tyson GW, Webster N (2013) Coral reef invertebrate microbiomes correlate with the presence of photosymbionts Isme J 7(7):1452-1458

59. Forget NL, Kim Juniper S (2013) Free-living bacterial communities associated with tubeworm (Ridgeia piscesae) aggregations in contrasting diffuse flow hydrothermal vent habitats at the Main Endeavour Field, Juan de Fuca Ridge MicrobiologyOpen 2(2): 259-275

60. Dishaw LJ, Flores-Torres J, Lax S, Gemayel K, Leigh B, Melillo D, Mueller MG, Natale L, Zucchetti I, De Santis R, Pinto MR, Litman GW, Gilbert JA (2014) The gut of geographically disparate Ciona intestinalis harbors a core microbiota PLoS One 9(4):e93386 
61. Nishijima M, Adachi K, Katsuta A, Shizuri Y, Yamasato K (2013) Endozoicomonas numazuensis sp. nov., a gammaproteobacterium isolated from marine sponges, and emended description of the genus Endozoicomonas Kurahashi and Yokota 2007 Int J Syst Evol Microbiol 63(Pt 2):709-714

62. Neave MJ, Rachmawati R, Xun L, Michell CT, Bourne DG, Apprill A, Voolstra CR (2016) Differential specificity between closely related corals and abundant Endozoicomonas endosymbionts across global scales Isme J 11(1):186-200

63. Sussman M, Willis BL, Victor S, Bourne DG (2008) Coral pathogens identified for white syndrome (WS) epizootics in the IndoPacific PLoS One 3(6):e2393

64. Ben-Haim Y, Zicherman-Keren M, Rosenberg E (2003) Temperature-regulated bleaching and lysis of the coral Pocillopora damicornis by the novel pathogen Vibrio coralliilyticus Appl Environ Microbiol 69(7):4236-4242

65. Kushmaro A, Rosenberg E, Fine M, Loya Y (1997) Bleaching of the coral Oculina patagonica by Vibrio AK-1 Mar Ecol Prog Ser 147:159-165

66. Rubio-Portillo E, Yarza P, Penalver C, Ramos-Espla AA, Anton J (2014) New insights into Oculina patagonica coral diseases and their associated Vibrio spp. communities Isme J 8(9):1794-1807

67. Holm JB, Heidelberg KB (2016) Microbiomes of Muricea californica and M. fruticosa: comparative analyses of two cooccurring eastern Pacific octocorals Front Microbiol 7 (917)

68. Gray MA, Stone RP, McLaughlin MR, Kellogg CA (2011) Microbial consortia of gorgonian corals from the Aleutian islands FEMS Microbiol Ecol 76(1):109-120

69. Penn K, Wu D, Eisen JA, Ward N (2006) Characterization of bacterial communities associated with deep-sea corals on Gulf of Alaska seamounts Appl Environ Microbiol 72(2):1680-1683

70. Kellogg CA, Lisle JT, Galkiewicz JP (2009) Culture-independent Characterization of bacterial communities associated with the coldwater coral Lophelia pertusa in the northeastern Gulf of Mexico Appl Environ Microbiol 75(8):2294-2303

71. Neulinger SC, Gärtner A, Järnegren J, Ludvigsen M, Lochte K, Dullo W-C (2009) Tissue-associated "Candidatus Mycoplasma corallicola" and filamentous bacteria on the cold-water coral Lophelia pertusa (Scleractinia) Appl Environ Microbiol 75(5): $1437-1444$

72. Mortzfeld BM, Urbanski S, Reitzel AM, Kunzel S, Technau U, Fraune S (2016) Response of bacterial colonization in Nematostella vectensis to development, environment and biogeography Environ Microbiol 18(6):1764-1781

73. Wang Y, Stingl U, Anton-Erxleben F, Geisler S, Brune A, Zimmer M (2004) "Candidatus Hepatoplasma crinochetorum," a new, stalk-forming lineage of Mollicutes colonizing the midgut glands of a terrestrial isopod Appl Environ Microbiol 70(10):6166-6172

74. Fraune S, Zimmer M (2008) Host-specificity of environmentally transmitted Mycoplasma-like isopod symbionts Environ Microbiol 10(10):2497-2504

75. Fraune S, Bosch TCG (2007) Long-term maintenance of speciesspecific bacterial microbiota in the basal metazoan Hydra Proc Natl Acad Sci U S A 104(32):13146-13151

76. Matsuura Y, Kikuchi Y, Meng XY, Koga R, Fukatsu T (2012) Novel clade of alphaproteobacterial endosymbionts associated with stinkbugs and other arthropods Appl Environ Microbiol 78(12): 4149-4156

77. Ziegler M, Seneca FO, Yum LK, Palumbi SR, Voolstra CR (2017) Bacterial community dynamics are linked to patterns of coral heat tolerance Nat Commun 8:14213
78. Walmsley TA, Matcher GF, Zhang F, Hill RT, Davies-Coleman MT, Dorrington RA (2012) Diversity of bacterial communities associated with the Indian Ocean sponge Tsitsikamma favus that contains the bioactive pyrroloiminoquinones, tsitsikammamine $\mathrm{A}$ and $\mathrm{B}$ Mar Biotechnol (New York, NY) 14(6):681-691

79. Israelsson $O$ (2007) Chlamydial symbionts in the enigmatic Xenoturbella (Deuterostomia) J Invertebr Pathol 96(3):213-220

80. Li J, Chen Q, Long L-J, Dong J-D, Yang J, Zhang S (2014) Bacterial dynamics within the mucus, tissue and skeleton of the coral Porites lutea during different seasons Sci Rep 4:7320

81. Chen C-P, Tseng C-H, Chen CA, Tang S-L (2011) The dynamics of microbial partnerships in the coral Isopora palifera Isme J 5(4): 728-740

82. Ceh J, Van Keulen M, Bourne DG (2011) Coral-associated bacterial communities on Ningaloo Reef, Western Australia FEMS Microbiol Ecol 75(1):134-144

83. Kimes NE, Johnson WR, Torralba M, Nelson KE, Weil E, Morris PJ (2013) The Montastraea faveolata microbiome: ecological and temporal influences on a Caribbean reef-building coral in decline Environ Microbiol 15(7):2082-2094

84. Meistertzheim A-L, Lartaud F, Arnaud-Haond S, Kalenitchenko D, Bessalam M, Le Bris N, Galand PE (2016) Patterns of bacteria-host associations suggest different ecological strategies between two reef building cold-water coral species Deep-Sea Res I Oceanogr Res Pap 114:12-22

85. La Rivière M, Roumagnac M, Garrabou J, Bally M (2013) Transient shifts in bacterial communities associated with the temperate gorgonian Paramuricea clavata in the Northwestern Mediterranean Sea PLoS One 8(2):e57385

86. Bourne D, Iida Y, Uthicke S, Smith-Keune C (2008) Changes in coral-associated microbial communities during a bleaching event Isme J 2(4):350-363

87. Cocito S, Bedulli D, Sgorbini S (2002) Distribution patterns of the sublittoral epibenthic assemblages on a rocky shoal in the Ligurian Sea (NW Mediterranean) Sci Mar 66(2):175-181

88. Frias-Lopez J, Zerkle AL, Bonheyo GT, Fouke BW (2002) Partitioning of bacterial communities between seawater and healthy, black band diseased, and dead coral surfaces Appl Environ Microbiol 68(5):2214-2228

89. Sekar R, Kaczmarsky L, Richardson L (2008) Microbial community composition of black band disease on the coral host Siderastrea siderea from three regions of the wider Caribbean Mar Ecol Prog Ser 362:85-98

90. Ruehland C, Blazejak A, Lott C, Loy A, Erséus C, Dubilier N (2008) Multiple bacterial symbionts in two species of cooccurring gutless oligochaete worms from Mediterranean sea grass sediments Environ Microbiol 10(12):3404-3416

91. Brune A (2014) Symbiotic digestion of lignocellulose in termite guts Nat Rev Microbiol 12(3):168-180

92. Sally AM, Smucker RA (1987) Role of Cristispira sp. and other bacteria in the chitinase and chitobiase activities of the crystalline style of Crassostrea virginica (Gmelin) Microb Ecol 14(2):157-166

93. Harris JK, Kelley ST, Pace NR (2004) New perspective on uncultured bacterial phylogenetic division OP11 Appl Environ Microbiol 70(2):845-849

94. Nelson W, Stegen J (2015) The reduced genomes of Parcubacteria (OD1) contain signatures of a symbiotic lifestyle Front Microbiol6 (713)

95. Rosenberg E, Koren O, Reshef L, Efrony R, Zilber-Rosenberg I (2007) The role of microorganisms in coral health, disease and evolution Nat Rev Microbiol 5(5):355-362 